\title{
Comorbidities and treatment patterns in adult patients with atopic dermatitis: results from a nationwide multicenter study
}

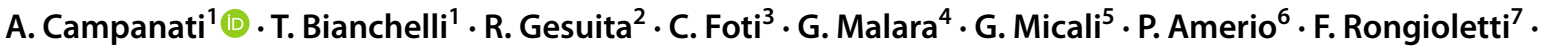

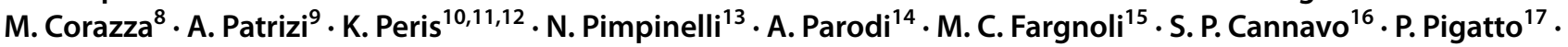 \\ G. Pellacani ${ }^{18} \cdot$ S. M. Ferrucci ${ }^{19,20} \cdot$ G. Argenziano ${ }^{21} \cdot$ F. Cusano ${ }^{22} \cdot$ G. Fabbrocini ${ }^{23} \cdot$ L. Stingeni $^{24} \cdot$ M. C. Potenza ${ }^{25}$. \\ M. Romanelli ${ }^{26} \cdot$ L. Bianchi $^{27} \cdot$ A. Offidani ${ }^{1}$ and collaborators
}

Received: 2 October 2020 / Revised: 15 February 2021 / Accepted: 18 March 2021 / Published online: 7 June 2021

(c) The Author(s) 2021, corrected publication 2021

\begin{abstract}
Adult atopic dermatitis (adult AD) is a systemic inflammatory disorder, whose relationship with immune-allergic and metabolic comorbidities is not well established yet. Moreover, treatment of mild-to-moderate and severe atopic dermatitis needs standardization among clinicians. The aim of this study was to evaluate the distribution of comorbidities, including metabolic abnormalities, rhinitis, conjunctivitis, asthma, alopecia and sleep disturbance, according to severity of adult AD, and describe treatments most commonly used by Italian dermatologists. Retrospective, observational, nationwide study of adult patients over a 2-year period was performed. Clinical and laboratory data were obtained through review of medical records of patients aged $\geq 18$ years, followed in 23 Italian National reference centres for atopic dermatitis between September 2016 and September 2018. The main measurements evaluated were disease severity, atopic and metabolic comorbidities, treatment type and duration. Six-hundred and eighty-four adult patients with AD were included into the study. Atopic, but not metabolic conditions, except for hypertension, were significantly associated with having moderate-to-severe AD in young adult patients. Disease duration was significantly associated with disease severity. Oral corticosteroids and cyclosporine were the most widely used immunosuppressant. Our study seems confirm the close relationship between adult AD and other atopic conditions, further long-term cohort studies on patients affected by adult AD need to be performed to evaluate the complex relationship between adult $\mathrm{AD}$ disease severity and metabolic comorbidities.
\end{abstract}

Keywords Adult atopic dermatitis $\cdot$ Epidemiology $\cdot$ Comorbidity $\cdot$ Treatment $\cdot$ Immunosuppressants $\cdot$ Biologics

\section{Introduction}

Atopic dermatitis (AD) usually appears in early childhood $(15-30 \%)$ and generally resolves prior to puberty. However, in up to half of patients, it may persist into adulthood, becoming a lifelong condition $[1,2]$.

Although prevalence of adult atopic dermatitis (adult AD) remains unclear, several studies have indicated that it has been increasing in recent decades, particularly in industrialized countries [3]. Current estimates place the prevalence of $\mathrm{AD}$ at around $2-8 \%$ in adults, compared with $10-20 \%$ in children $[4,5]$.

Collaborators are listed under Acknowledgments.

A. Patrizi

annalisa.patrizi@unibo.it

Extended author information available on the last page of the article
Adult AD is considered a systemic and immune-allergic inflammatory skin disease, and it was assumed that atopic (allergic rhinitis, asthma), metabolic comorbidities (increased body mass index (BMI), central obesity, hypertension, hyperlipidaemia, diabetes mellitus) and sleep disturbance can promote the chronic inflammatory state, capable of perpetuating the progression of the disease, leading to more severe forms of adult $\mathrm{AD}[6,7]$.

Topical and systemic corticosteroids are the cornerstone of pharmacological treatment regardless disease severity [8, 9].

However, in moderate and severe cases, several systemic treatments are used in clinical practice, including cyclosporine, which is the only one approved for treatment of adult $\mathrm{AD}$, followed by methotrexate, azathioprine, and mycophenolate mofetil. 
There are still no population-based Italian studies on the prevalence of comorbidities in adult $\mathrm{AD}$ based on the severity of disease.

Similarly, the need for observational studies describing therapeutic interventions that reflect the impact on these patients is growing. Aims of this study were to evaluate the distribution of associated adult AD comorbidities according to disease severity, through the stratification of patients with mild, moderate and severe disease, and describe treatments prescribed for adult patients in routine clinical practice, according to disease severity.

\section{Materials and methods}

\section{Study design}

This is a multicenter observational study, conducted in accordance with the latest revision of the Helsinki Declaration (2009/58). All enrolled patients had signed an informed consent to make their clinical data available for research purposes. The study was approved by Local Ethical Committee United Hospital of Ancona.

\section{Population}

Data from 684 patients (356 males and 328 females) with mild-to-severe atopic dermatitis, aged $\geq 18$ years, in treatment with conventional topical and systemic drugs at 23 Dermatological clinical centres of Italian National Health System were retrospectively collected from outpatients and inpatients medical records from September 2016 to September 2018 and recorded into an electronic medical record.

\section{Clinical collected data}

Information such as demographic data (age and sex), anthropometric measurements (height, weight and BMI, waist and hip circumference), metabolic (hypertriglyceridemia, hypercholesterolemia, hypertension, diagnosis of type 2 diabetes mellitus), atopic (rhinitis, asthma, conjunctivitis) comorbidities and functional impairment as sleep disorders (including difficulty falling asleep, frequent night time awakenings, and excessive daytime sleepiness) and pruritus were retrieved. Previous and on-going treatments for each patient were collected as well.

\section{Clinimetric tools}

Severity of adult AD for each subject was recorded as mild, moderate or severe, according to Eczema Area and Severity Index (EASI) and SCORing AD (SCORAD). Quality of life of patients were investigated through the Dermatology Life Quality Index (DLQI) questionnaire.

EASI is a validated investigator-assessed scoring system that grades the physical signs of atopic dermatitis, it determines the severity of the patient's eczema, according to clinicians' perspective [10], its final score ranges from 0 to 72 .

SCORAD index is a mixed patient/clinicians tool to evaluate $A D$ severity [11], its final score ranges from 0 to 103 , and determines disease severity according to patients and clinicians' perspective.

Diagnosis and disease severity of atopic dermatitis was established according Italian AD guidelines [12], AD diagnosis was based essentially on the disease typical clinical signs and symptoms, evaluated by experienced dermatologists, as currently no diagnostic markers are available. Disease severity was graduated as follows: mild disease EASI $<16$ and/or SCORAD $<25$; moderate disease $\mathrm{EASI}=>16$ and/or $\mathrm{SCORAD}>25$; severe disease EASI $=>21$ and/or SCORAD $>50$, or EASI score $<16$ but at least one of the following conditions: itch with numeric rating scale (NRS) score $>7$ and/or sleep disturbances with NRS score $>7$.

DLQI is a simple, self-administered and user-friendly validated questionnaire designed to assess the impact of a wide range of skin disease on patient health-related quality of life [13]. Final total score is calculated by summing the score of ten items which cover six domains including symptoms and feelings, daily activities, leisure, work and school, personal relationships, resulting in a maximum score of 30 and a minimum score of 0 . The higher scores the poorer the quality of life.

Pruritus was quantified through The Peak Pruritus Numerical Rating Scale (NRS) a validated, single-item, patient-reported outcome (PRO) of itch severity. Patients were asked to rate the intensity of their itch using a visual analogue scale rating from 0 ("no itch") to 10 ("worst imaginable itch").

\section{Statistical analysis:}

A non-parametric approach was used since variables had a no-normal distribution, when evaluated with the Shapiro test. Quantitative variables were summarized using median and interquartile range (IQR, $1^{\text {st }}-3$ rd quartiles), respectively, as measure of centrality and variability; qualitative 
variables were expressed as absolute and percent frequencies. Comparisons between groups were evaluated using Wilcoxon rank sum test and Fisher exact test, respectively.

A logistic regression analysis was performed to evaluate demographic and clinical factors associated with the probability of having a severe vs mild/moderate AD.

Likelihood Ratio (LR) test was used to detect the variables to be included in the model and models' goodness of fit was evaluated by the Hosmer and Lemeshow (HL) test. All estimates were evaluated as $95 \%$ confidence intervals (95\% CI).

\section{Results}

A total of 684 adult patients with $\mathrm{AD}$ were included in this study, among which $79(11.5 \%)$ were classified to have mild, $71(10.5 \%)$ moderate, and 534 (78\%) severe AD. Distribution of demographic, anthropometric and clinical characteristics of the patients according to adult AD severity is summarized in Table 1.
Patients having severe diseases were significantly older than the other $(p=0.05)$, and no difference in adult AD severity according to sex between groups was evident $(p=0.212)$.

Median disease duration ranging from 18 years (IQR: 6-27 for mild adult AD, and 7-27 for moderate adult AD) to 23 years (IQR: 15-35) for severe $\mathrm{AD}$, with significantly longer lasting disease for patients having severe adult $\mathrm{AD}$ $(p<0.001)$.

Impact of adult $\mathrm{AD}$ on quality of life was significantly different across the three groups, with DLQI gradually increasing from mild 4 (IQR: 2-6) to moderate 7 (IQR: 4-12) and severe 16 (IQR: $11-21)$ atopic dermatitis $(p<0.001)$.

Pruritus (NRS peak pruritus) showed the same pattern, and it was more pronounced in severe Adult AD (8; IQR: 7-10), compared to moderate (5; IQR: 2-7) and mild (3; IQR: 2-5).

Among metabolic features (Tables 1and2), hypertension was found significantly more frequent in the severe adult AD group $(p<0.001)$; no significant difference was retrieved across the three groups for: BMI $(p=0.301)$, abdominal obesity (considered as $\mathrm{W} / \mathrm{H}>0.9$ for males and 0.85 for females;

Table 1 Demographic, anthropometric and clinical characteristics of the patients according to adult AD severity

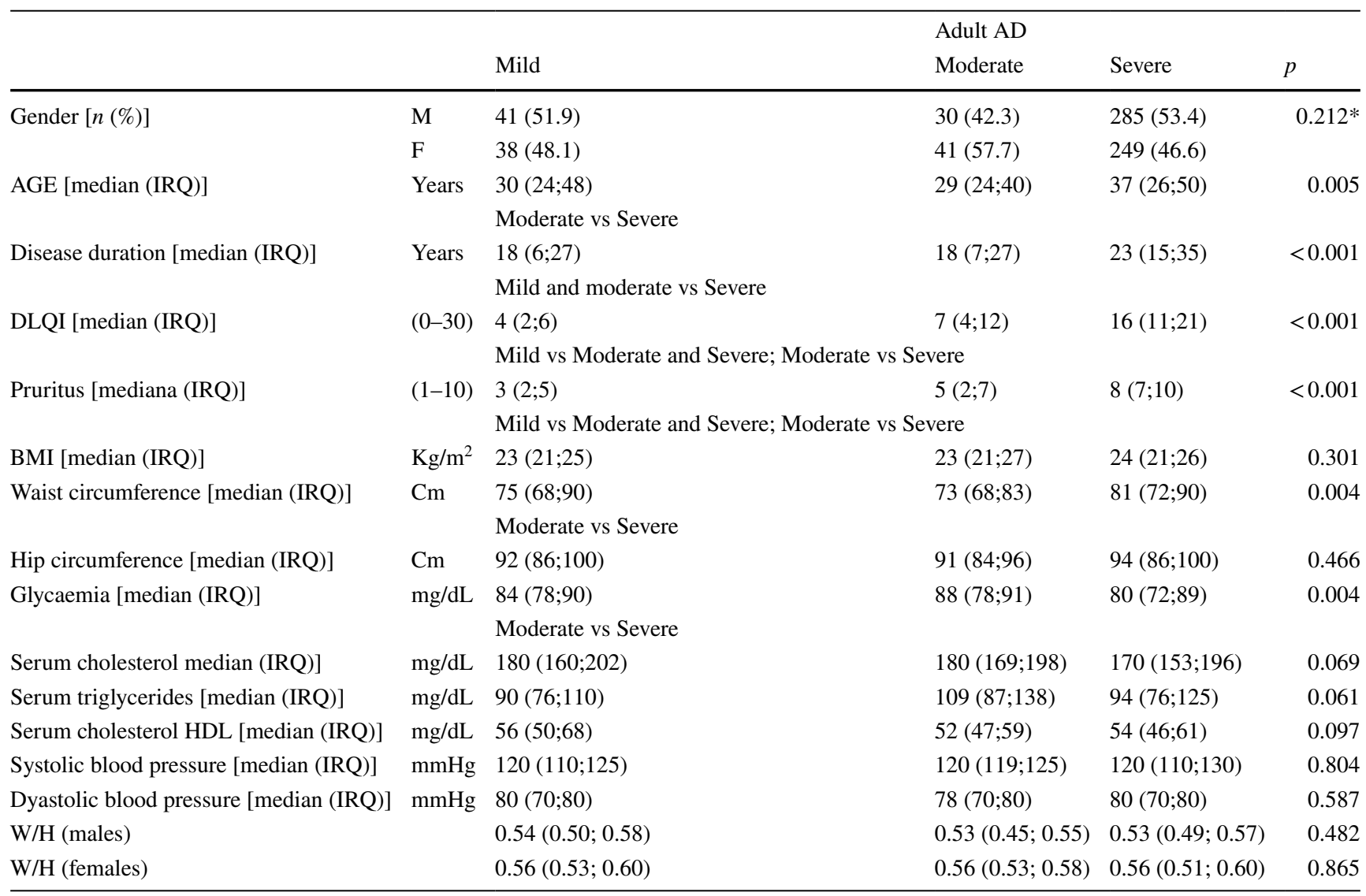

$p$ values refer to Kruskal-Wallis for quantitative variables; IRQ: $1^{\circ}-3^{\circ}$ quartiles

*Chi-square test for categorical variables 
Table 2 Metabolic comorbidities according to adult AD severity

\begin{tabular}{|c|c|c|c|c|c|c|}
\hline & & \multicolumn{3}{|l|}{ Adult $\mathrm{AD}$} & \multirow[b]{2}{*}{$n=684$} & \multirow[b]{2}{*}{$p$} \\
\hline & & $\begin{array}{l}\text { Mild } \\
n=79\end{array}$ & $\begin{array}{l}\text { Moderate } \\
n=71\end{array}$ & $\begin{array}{l}\text { Severe } \\
n=534\end{array}$ & & \\
\hline & & $n=69$ & $n=62$ & $n=378$ & $n=509$ & \\
\hline \multirow[t]{3}{*}{ Hypercholesterolemia $[n(\%)]$} & No & $51(73.9)$ & $48(77.4)$ & $298(78.8)$ & & 0.658 \\
\hline & Sì & $18(26.1)$ & $14(22.6)$ & $80(21.2)$ & $112(22)$ & \\
\hline & & $n=69$ & $n=62$ & $n=374$ & $n=505$ & \\
\hline \multirow[t]{3}{*}{ Hypertriglyceridemia $[n(\%)]$} & No & $67(97.1)$ & $60(96.8)$ & $367(98.1)$ & & 0.602 \\
\hline & Sì & $2(2.9)$ & $2(3.2)$ & $7(1.9)$ & $11(2.2)$ & \\
\hline & & $n=70$ & $n=61$ & $n=370$ & $n=501$ & 0.841 \\
\hline \multirow[t]{3}{*}{ Diabetes $[n(\%)]$} & No & 69 (98.6) & $61(100)$ & $364(98.4)$ & & \\
\hline & Sì & $1(1.4)$ & $(0)$ & $6(1.6)$ & $7(1.4)$ & \\
\hline & & $n=56$ & $n=50$ & $n=304$ & $n=410$ & 0.999 \\
\hline \multirow[t]{3}{*}{ Abdominal obesity $[n(\%)]$} & No & $56(100)$ & $50(100)$ & $302(99.3)$ & & \\
\hline & Sì & $(0)$ & $(0)$ & $2(0.7)$ & $2(0.5)$ & \\
\hline & & $n=77$ & $n=68$ & $n=393$ & $n=538$ & $<0.001$ \\
\hline \multirow[t]{3}{*}{ Hypertension $[n(\%)]$} & No & $69(89.6)$ & $68(100)$ & $333(84.7)$ & & \\
\hline & Sì & $8(10.4)$ & $(0)$ & $60(15.3)$ & $68(12.6)$ & \\
\hline & & $n=68$ & $n=60$ & $n=358$ & $n=486$ & 0.707 \\
\hline \multirow[t]{2}{*}{ Metabolic syndrome $[n(\%)]$} & No & $67(98.5)$ & $60(100)$ & $355(99.2)$ & & \\
\hline & Sì & $1(1.5)$ & $(0)$ & $3(0.8)$ & $4(0.8)$ & \\
\hline
\end{tabular}

$p$ values refer to Fisher exact test $p=0.999)$, hypercholesterolemia $(p=0.658)$, hypertriglyceridemia $(p=0.602)$, diabetes mellitus $(p=0.841)$.

Atopic comorbidities showed a significant association to adult $\mathrm{AD}$ severity, with frequencies increasing from mild to moderate and severe adult $\mathrm{AD}$ (asthma $p<0.001$; conjunctivitis $p<0.001$; rhinitis $p=0.002$; alopecia $p<0.001)$. Similarly, sleep disorders, were more frequent among patients suffering from severe, rather than mild or moderate adult $\mathrm{AD}(p<0.001)$ (Table 3$)$.
Table 3 Atopic comorbidities according to adult AD severity

\begin{tabular}{lllllll}
\hline & \multicolumn{3}{l}{ Adult AD } & & \\
\cline { 2 - 4 } & & Mild & Moderate & Severe & & \\
& & $n=79$ & $n=71$ & $n=534$ & $n=684$ & \\
\hline Asthma $[n(\%)]$ & No & $62(78.5)$ & $53(74.6)$ & $336(62.9)$ & & $<0.001$ \\
Conjunctivitis $[n(\%)]$ & No & $58(73.4)$ & $50(70.4)$ & $290(54.3)$ & & \\
& Yes & $21(26.6)$ & $21(29.6)$ & $244(45.7)$ & $286(41.8)$ & \\
Rhinitis $[n(\%)]$ & No & $46(58.2)$ & $35(49.3)$ & $207(38.8)$ & & 0.001 \\
& Yes & $33(41.8)$ & $36(50.7)$ & $327(61.2)$ & $396(57.9)$ & \\
Sleep disorders $[n(\%)]$ & No & $64(81)$ & $43(60.6)$ & $163(30.5)$ & & $<0.001$ \\
& Yes & $15(19)$ & $28(39.4)$ & $371(69.5)$ & $414(60.5)$ & \\
Other disturbs $[n(\%)]$ & & $n=67$ & $n=51$ & $n=470$ & $n=588$ & \\
& No & $64(95.5)$ & $50(98)$ & $428(91.1)$ & & 0.136 \\
& Yes & $3(4.5)$ & $1(2)$ & $42(8.9)$ & $46(7.8)$ & \\
Alopecia $[n(\%)]$ & & $n=67$ & $n=51$ & $n=469$ & $n=587$ & $<0.001$ \\
& No & $65(97)$ & $37(72.5)$ & $431(91.9)$ & & \\
& Yes & $2(3)$ & $14(27.5)$ & $38(8.1)$ & $54(9.2)$ & \\
\hline
\end{tabular}

$p$ values refer to Fisher exact test 
Table 4 Disease duration (years) according to atopic and metabolic comorbidities

\begin{tabular}{llll}
\hline & Absent & Present & $p$ \\
\hline Atopic comorbidities & & & \\
Asthma & $20(10-30)$ & $26(17-37)$ & $<0.001$ \\
Conjunctivitis & $20(9-30)$ & $25(17-36)$ & $<0.001$ \\
Rhinitis & $20(8-30)$ & $24(15-34)$ & $<0.001$ \\
Metabolic comorbidities & & & \\
Hypercholesterolemia & $20(10-29)$ & $24(10-40)$ & 0.039 \\
Hypertension & $20(10-29)$ & $27(8.5-40)$ & 0.182 \\
Hypertriglyceridemia & $20(10-30)$ & $30(14-53)$ & 0.111 \\
\hline
\end{tabular}

Values are medians (1st-3rd quartiles). $p$ values refer to Wilcoxon rank sum test
Disease duration was significantly longer in the presence of all the atopic comorbidities (Table 4), whereas among metabolic comorbidities, only hypercholesterolemia was associated with a longer disease duration.

As regards topical treatments (Table 5), the majority part of patients $(98.3 \%)$ was under treatment or had received topical corticosteroids, at least once in their life $(98.7 \%$ of patients with mild adult $\mathrm{AD}, 97.1 \%$ of patients with moderate adult $\mathrm{AD}$, and $98.5 \%$ of patients with severe adult $\mathrm{AD}$ had experience of treatment with topical corticosteroids) with an increasing trend, moving from mild to moderate and severe $\operatorname{AD}(p=0.003)$.

The use of topical calcineurin inhibitors was less frequent than corticosteroids (only $32.8 \%$ of the patients was receiving or had received them in the past), among them $63.3 \%$ had mild, $56.3 \%$ moderate, and $25.3 \%$ had severe adult AD.

The trend of resorting to calcineurin inhibitors increased with the worsening of disease $(p<0.001)$. Over
Table 5 Topical and systemic treatments according to disease severity

\begin{tabular}{|c|c|c|c|c|c|}
\hline & & \multicolumn{3}{|l|}{ Adult AD } & \multirow[b]{2}{*}{$p$} \\
\hline & & $\begin{array}{l}\text { Mild } \\
n=79\end{array}$ & $\begin{array}{l}\text { Moderate } \\
n=71\end{array}$ & $\begin{array}{l}\text { Severe } \\
n=534\end{array}$ & \\
\hline \multirow[t]{3}{*}{ Topical corticosteroids $[n(\%)]$} & Never & $1(1.3)$ & $2(2.8)$ & $8(1.5)$ & 0.003 \\
\hline & Previous & $58(73.4)$ & $41(57.7)$ & $276(51.7)$ & \\
\hline & On-going & $20(25.3)$ & $28(39.4)$ & $250(46.8)$ & \\
\hline \multirow{3}{*}{$\begin{array}{l}\text { Topical calcineurin inhibitors } \\
{[n(\%)]}\end{array}$} & Never & $50(63.3)$ & $40(56.3)$ & $135(25.3)$ & $<0.001$ \\
\hline & Previous & $21(26.6)$ & $21(29.6)$ & $287(53.7)$ & \\
\hline & On-going & $8(10.1)$ & $10(14.1)$ & $112(21)$ & \\
\hline \multirow[t]{3}{*}{ Systemic corticosteroids $[n(\%)]$} & Never & $26(32.9)$ & $20(28.2)$ & $80(15)$ & $<0.001$ \\
\hline & Previous & $50(63.3)$ & $48(67.6)$ & $404(75.7)$ & \\
\hline & On-going & $3(3.8)$ & $3(4.2)$ & $50(9.4)$ & \\
\hline \multirow[t]{4}{*}{ Systemic antihistamines $[n(\%)]$} & Never & $12(15.2)$ & $13(18.3)$ & $30(5.6)$ & $<0.001$ \\
\hline & Previous & $52(65.8)$ & $43(60.6)$ & $360(67.4)$ & \\
\hline & On-going & $15(19)$ & $15(21.1)$ & $144(27)$ & \\
\hline & & $n=79$ & $n=71$ & $n=531$ & \\
\hline \multirow[t]{3}{*}{ Puva/uva/uvbnb $[n(\%)]$} & Never & $65(82.3)$ & $59(83.1)$ & $297(55.9)$ & $<0.001$ \\
\hline & Previous & $11(13.9)$ & $10(14.1)$ & $218(41.1)$ & \\
\hline & On-going & $3(3.8)$ & $2(2.8)$ & $16(3)$ & \\
\hline \multirow[t]{3}{*}{ Cyclosporine A $[n(\%)]$} & Never & $49(62)$ & $46(64.8)$ & $149(27.9)$ & $<0.001$ \\
\hline & Previous & $27(34.2)$ & $18(25.4)$ & $329(61.6)$ & \\
\hline & On-going & $3(3.8)$ & $7(9.9)$ & $56(10.5)$ & \\
\hline \multirow[t]{3}{*}{ Metotrexate $[n(\%)]$} & Never & $77(97.5)$ & $67(94.4)$ & $475(89)$ & 0.106 \\
\hline & Previous & $2(2.5)$ & $4(5.6)$ & $52(9.7)$ & \\
\hline & On-going & $0(0)$ & $0(0)$ & $7(1.3)$ & \\
\hline \multirow[t]{3}{*}{ Azathioprine $[n(\%)]$} & Never & $79(100)$ & $69(97.2)$ & $510(95.5)$ & 0.386 \\
\hline & Previous & $0(0)$ & $2(2.8)$ & $22(4.1)$ & \\
\hline & On-going & $0(0)$ & $0(0)$ & $2(0.4)$ & \\
\hline \multirow{2}{*}{$\begin{array}{l}\text { Mycophenolate } \\
\text { mofetil/sodium }[n(\%)]\end{array}$} & Never & $79(100)$ & $71(100)$ & $533(99.8)$ & 0.386 \\
\hline & Previous & $0(0)$ & $0(0)$ & $1(0.2)$ & \\
\hline
\end{tabular}

Fisher exact test 
half of the patients (61.5\%), regardless disease severity, had not received any phototherapy treatment (PUVA, UVA, UVB, UVB nb), for all the duration of their disease $(82.3 \%$ of patients with mild, $83.1 \%$ with moderate and $55.9 \%$ with severe adult $\mathrm{AD}$ ). The use of phototherapy was significantly more frequent in patients with severe adult $\mathrm{AD}$, compared to those with mild or moderate adult $\mathrm{AD}(p<0.001)$.

For what concerns to systemic treatments (Table 4), the majority of patients $(81.5 \%)$ had been treated with systemic corticosteroids in the past, or they were still on treatment $(67.1 \%$ of patients with mild, $71.8 \%$ moderate, and $85.1 \%$ severe $\mathrm{AD}$ ), the use of systemic corticosteroids increases in parallel with the worsening of disease $(p<0.001)$.

Conversely, the majority of patients with mild (62\%) and moderate (64.8) adult $\mathrm{AD}$, had not received any cyclosporine treatment throughout their disease duration, whereas $72.1 \%$ of patients with severe adult AD had been treated in the past or they were still in treatment with cyclosporine. Thus, the use of cyclosporine was significantly more frequent in patients with severe adult AD, compared to mild and moderate adult $\operatorname{AD}(p<0.001)$.

In general, long-term treatment with oral immunosuppressive therapy was usually introduced when topical treatment with mid- to high-potent corticosteroids and/ or calcineurin inhibitors had not been successful. Among immunosuppressive drugs, Cyclosporine was the most widely used agent, for a minimum treatment period of 3 months and no longer than 2 years. Median period of cyclosporin treatment was $6.5 \pm 2.8$ month. Main reasons for cyclosporin discontinuation included: disease control in $25.6 \%$ of patients, adverse events in $21.2 \%$ of patients, ineffectiveness in $17.1 \%$, and adverse events plus ineffectiveness in $5.7 \%$ of enrolled patients.

As regards other immunosuppressive agents: $97.5 \%$ of patients with mild, $94.4 \%$ of patients with moderate, and $89 \%$ of patients with severe had never been treated with methotrexate; $100 \%$ of patients with mild, $97.7 \%$ of patients with moderate, and $95.5 \%$ of patients with mild had not received azathioprine; only one patient with severe disease had had experience of treatment with mycophenolate mofetil. No difference among categories of disease severity was evident for use of immunosuppressive agents other than cyclosporine $(p=0.386)$.

Table 6 shows the results of logistic regression analysis. Disease duration and hypertension were found significantly associated with severe adult AD. In particular, the risk of having a severe adult AD increased of $2 \%$ for every year of disease duration added, and 3.52 time in presence of hypertension.
Table 6 Factors associated to adult AD severity

\begin{tabular}{llll}
\hline Factors & OR & $95 \% \mathrm{CI}$ & $p$ \\
\hline Disease duration (years) & 1.02 & $1.01-1.04$ & 0.006 \\
Asthma (yes vs no) & 1.41 & $0.82-2.47$ & 0.215 \\
Conjunctivitis (yes vs no) & 1.56 & $0.87-2.81$ & 0.135 \\
Rhinitis (yes vs no) & 1.05 & $0.61-1.83$ & 0.853 \\
Hypercholesterolemia (yes vs no) & 0.66 & $0.40-1.10$ & 0.108 \\
Hypertension (yes vs no) & 3.52 & $1.62-8.85$ & 0.003 \\
\hline
\end{tabular}

Results from logistic regression. LR test: $\chi^{2}=30.7, \mathrm{df}=6, p$ value $<0.001 ; \mathrm{HL}$ test: $\chi^{2}=6.98, \mathrm{df}=8, p$ value 0.539

\section{Discussion}

In the last decade, the relationships between chronic cutaneous and systemic diseases have emerged as major clinical, public health and research issues. Consequently, clinical and epidemiological researches focusing on comorbidities of skin diseases are currently recognized as one of the most important tools to indirectly increase knowledge on their physiopathology and to profile the burden of disease.

The most notable finding of our study is the confirmation of a significant association between atopic diseases like asthma, conjunctivitis, alopecia and rhinitis, and adult AD severity in Italian population through a nationwide study.

This is consistent with several data recently merging from the literature. In 2019, Kok et al. [7] reported that a dosedependent effect can be found between the association of atopic comorbidities and severity of adult $\mathrm{AD}$, which suggests that chronic severe adult AD may result in increased disease burden and morbidity.

Similarly, Sicras-Mainar A et al. [14] in 2019 described a close association between adult AD severity and other immune-allergic expressions such as asthma and rhinitis.

These data must be interpreted in the perspective that the overall prognosis for patients with one or more atopic comorbidities has a worse clinical course compared to patients suffering from atopic dermatitis alone $[8,9]$.

This evidence is reinforced by the observation of Thijs et al. [15] who reported that adult AD with coexistent atopic conditions is associated with more severe and extensive disease. The authors explain these associations through the increased expression of several serum pro-inflammatory mediators like PARC, TIMP-1 and sCD14, and a great selective Th- 2 cytokine inflammatory pressure in this subset of patients.

These are actually expected events, if we refer to the pathogenic model of "atopic march", a temporal development model widely used in epidemiological studies to interpret the temporal changes in the prevalence of eczema, asthma, and allergic rhinitis [16, 17]. 
Definitive conclusions about temporality, and causal relationship between observed associations are impossible to be drawn, as most studies reported in the literature have a cross-sectional design. However, the existence of a common inflammatory pathogenic Th-2 pathway might explain the higher correlation between severity of adult $\mathrm{AD}$ and other atopic conditions, compared with metabolic abnormalities, as reported also by Kok et al. [7]. Indeed recently, a unifying hypothesis of a type 2 inflammation mechanism involving T Helper 2 responses has been suggested for all the comorbid atopic conditions (i.e. atopic asthma, AD, atopic conjunctivitis) [18].

The association between adult AD and metabolic abnormalities has been postulated on the basis of the "inflammatory skin march model," first identified in psoriasis patients with systemic inflammatory condition [19].

According to this model, Th1, Th17, and Th22 proinflammatory cytokines mediate their effects via binding to their own cytokine receptors and then activating several downstream pathways, driving the connection between metabolic syndrome and atopic dermatitis in adults [19]. However, association between metabolic abnormalities and adult AD has not been fully established yet [20].

The cross-sectional study of Kok et al. [7] conducted on 5007 Korean adults reported that metabolic syndrome, central obesity, and hypertriglyceridemia correlated positively with adult AD in women. However, Radtke et al. [21] in their cross-sectional analysis conducted on 37,456 patients with psoriasis, and 48,140 patients with adult AD demonstrated that, unlike the psoriasis cohort, the prevalence ratios for hypertension, hyperlipidaemia, and diabetes showed no difference in adult AD patients compared to non-adult $\mathrm{AD}$ patients.

Moreover, a recent systematic review by Ali et al. [22], which included 14 studies elucidating the significance of metabolic comorbidities in adult AD reported a positive association between adult AD and central obesity measured as waist circumference, and this association was stronger for women than men. The association between adult $\mathrm{AD}$ and hyperglycaemia appears unlikely, and inconsistent for hypercholesterolemia. Non-conclusive results can be traced for hypertension. However, the associations between hypertension, hyperglycaemia, cholesterol levels, and adult AD remain unclear, and central obesity could be the only component that correlates positively with Adult AD [21]. Moreover, other data from literature confirm obesity as the only metabolic parameter to be associated with increased prevalence and severity of Adult AD [23, 24].

Finally, Thyssen JP et al. [25] analyzed the 16 most relevant studies comparing cardiovascular risk factors and diabetes for adult patients with and without AD. No association was found between adult $\mathrm{AD}$ and type 2 diabetes, and hypertension. The authors conclude that it is unlikely that adult AD is, in itself, a risk factor for CVD [25].

In conclusion, it remains unclear whether adult $\mathrm{AD}$ is a risk factor for metabolic syndrome.

Our results show that hypertension is more frequent in severe adult AD patients and suffering from hypertension increases the risk of having a severe adult $\mathrm{AD}$ of more than 3.5 times. Moreover, in our population, very few people were found to be obese. This is not unexpected and confirms data from a recent systematic and meta-analysis review by Ascott et al. [26] who reported that significant associations with cardiovascular outcomes and adult AD were described in cohort studies, although no evidence was found among cross-sectional studies.

Moreover, observed associations between adult AD and cardiovascular diseases might have been confounded by poor health behaviors of patients with adult AD, such as smoking, reduced physical activity and drinking alcohol [25].

In general, epidemiologic data on adult $\mathrm{AD}$ comorbidities are rare and inconclusive due to the heterogeneity of study populations in terms of the studied outcomes, and absence of a gold standard.

There is a need for further epidemiologic studies, focusing on the prevalence of metabolic comorbidities according to disease severity. It must be indeed pointed out that the most part of large population cohort or database studies may not always take into account the severity of adult AD in their analyses.

Therapeutic management of signs and symptoms of adult AD with systemic anti-inflammatory and immunosuppressive agents has been widely described [14, 27].

Our results seem to be consistent with previous publications, agreeing that corticosteroids and cyclosporine stands out as the most used drugs in moderate-to-severe forms of adult $\mathrm{AD}$, among Italian Dermatologists.

Data from the reported cohort of patients reflect the Italian guidelines for the systemic treatment of AD: as systemic steroids have a largely unfavorable risk/benefit ratio for adult AD treatment, their long-term use in adult AD had not been generally recommended [12]. Short-term (up to 1-4 weeks) treatment had been considered a valid option only to treat an acute flare in severe cases of AD [12].

The only immunosuppressive agent with label indication for $\mathrm{AD}$ in Italy is cyclosporine, whose dosage can be easily personalized, in adults, based on the efficacy and tolerability in each individual patient [12]. Other immunosuppressive drugs, such as methotrexate and azathioprine, are used in the clinical practice though off-label, only when cyclosporine is contraindicated, not effective or not tolerated [12].

However, data on the efficacy of systemic treatments and the long-term safety of immunosuppressants are limited in adult $\mathrm{AD}$, thus further studies are needed to standardize the treatment approach. 
This is the first study aimed to evaluate the association of comorbidities with the severity of adult AD in Italian population. The strength of this study is the accurate selection of the sample size, consisting of a population cohort of 684 patients regularly diagnosed and followed at 23 national reference centres for atopic dermatitis. However, information retrospectively collected from medical records is measurably less accurate than information prospectively obtained, and for certain variables (e.g. abdominal obesity) about $30 \%$ of data were not available.

However, main limitation of our study consists in the retrospective nature of data collection, that is based on existing data recorded for reasons other than research. In this regard, it would have been of great interest to evaluate other comorbidities than those reported, unfortunately data focusing on lymphomas and other than hypertension cardiovascular diseases are not available. Similarly, incidence of use for each different UV and duration of treatment could be of interest, reflecting different approach in treatment of AD across different Italian regions. Unfortunately, details are not available in the reported cohort, and further observations in this field are warranted.

Moreover, in our case series, dupilumab was not included into the drugs evaluated, as it was not licensed and reimbursed by the Italian National Health System, at the moment of case collection. However, in the past few months, Dupilumab entered the therapeutic armamentarium of atopic dermatitis and other type 2 inflammatory disease. Many clinical trials on other biological agents and small molecules that may revolutionize the evolution and treatment of adult AD are on-going. Further studies are needed to define in more detail the clinical rationale behind the association between metabolic diseases and $\mathrm{AD}$, and to evaluate the potential effect of treatments in preventing comorbidities development in adult AD.

Acknowledgments The collaborators of this article are: P. Romita (Department of Biomedical Science and Human Oncology, Dermatological Clinic, University of Bari, Bari, Italy), M. L. Musumeci (Dermatology Clinic, University of Catania, PO G. Rodolico, AOU Policlinico-Vittorio Emanuele, Catania, Italy), V. Piras (Vita-Salute - S, Raffaele University, Milan, Italy), A. Borghi (Department of Medical Sciences, Dermatology Unit, University of Ferrara, Ferrara, Italy), C. Loi (Department of Experimental, Diagnostic and Specialty Medicine, Division of Dermatology, University of Bologna, Bologna, Italy), N. Gori (Fondazione Policlinico Universitario A. Gemelli IRCCS, Dermatologia, Rome, Italy; Sezione di Dermatologia, Dipartimento di Scienze Mediche e Chirurgiche, Università Cattolica del Sacro Cuore, Rome, Italy; Oncologia Medica, Università Cattolica del Sacro Cuore, Rome, Italy), F. Prigano (Department Health Science Section of Dermatology, University of Florence, Florence, Italy), R. Gallo (Dermatology Clinic, Ospedale Policlinico San Martino, Largo R. Benzi, 10, 16132 Genoa, Italy), M. Esposito (Dermatology, Department of Biotechnological and Applied Clinical Sciences, University of L'Aquila, L'Aquila, Italy), A. Campitello (Dermatology Unit, University Hospital Policlinico "G. Martino", Messina, Italy), L. Bolzano (Dermatology Unit, Department of Surgical, Medical, Dental and Morphological Science
With Interest Transplant, Oncological and Regenerative Medicine, University of Modena and Reggio Emilia, Modena, Italy), S. Tavecchio (Department of Physiopathology and Transplantation, Università Degli Studi Di Milano, Milan, Italy; Dermatology Unit, Fondazione IRCCS Ca' Granda Ospedale Maggiore Policlinico, Via Pace, 9, 20122 Milan, Italy), G. Calabrese (Dermatology Unit, University of Campania, Naples, Italy), L. Di Costanzo (Unit of Dermatology, G. Rummo Hospital, Benevento, Italy), K. Hansel (Dermatology Section, Department of Medicine, University of Perugia, Perugia, Italy), N. Skroza (Dermatology Unit, Department of Medico-Surgical Sciences and Biotechnologies, Faculty of Pharmacy and Medicine, Sapienza University of Rome - Polo Pontino, Rome, Italy), E. Tolino (Dermatology Unit, Department of Medico-Surgical Sciences and Biotechnologies, Faculty of Pharmacy and Medicine, Sapienza University of Rome - Polo Pontino, Rome, Italy), G. Tonini (Dermatology Unit, Department of Clinical and Experimental Medicine, University of Pisa, Pisa, Italy), M. Talamonti (Dermatology Unit, Policlinico Tor Vergata Rome, University of Rome Tor Vergata, Rome, Italy).

Funding Open access funding provided by Alma Mater Studiorum Università di Bologna within the CRUI-CARE Agreement.

\section{Declarations}

Conflict of interest Prof. Campanati A. speaker or board member for Abbvie, Galderma, Celgene, Leo-pharma, Eli Lilly, Merck Sharp and Dohme, Novartis, Pfizer, UCB. Prof. Amerio P. speaker and advisor for Abbvie, Eli Lilly, Galderma, Novartis, Sanofi-Genzyme, Janssen, Pfizer, Sandoz. Prof. Parodi A. speaker and advisor for Novartis, Almirall, Sanofi, Celgene, Amgen, Abbvie, Leo pharma, Lilly, Janssen, Pfizer, Galderma, Boehringer. Prof. Cannavò SP speaker or board member for Abbvie, Eli-Lilly, Janssen-Cilag, Leo Pharma, Novartis, Sanofi-Genzyme. Prof. Fargnoli MC speaker or board member for Almirall, Abbvie, Galderma, Leo Pharma, Mylan, Medac Pharma, Celgene, Pierre Fabre, UCB, Eli Lilly, Pfizer, Janssen, Novartis, Sanofi-Genzyme, Roche, Sunpharma, MSD. Prof. Potenza C, speaker and board member for, Almirall, Amgen, Abbvie, Celgene Leo pharma, Eli-Lilly, Janssen, Pfizer, Novartis, Sanofi- Genzyme. Prof. Offidani A, speaker or board member for Abbvie, Galderma, Celgene, Janssen, Leo-pharma, Eli Lilly, Merck Sharp and Dohme, Novartis, Pfizer, UCB.

Open Access This article is licensed under a Creative Commons Attribution 4.0 International License, which permits use, sharing, adaptation, distribution and reproduction in any medium or format, as long as you give appropriate credit to the original author(s) and the source, provide a link to the Creative Commons licence, and indicate if changes were made. The images or other third party material in this article are included in the article's Creative Commons licence, unless indicated otherwise in a credit line to the material. If material is not included in the article's Creative Commons licence and your intended use is not permitted by statutory regulation or exceeds the permitted use, you will need to obtain permission directly from the copyright holder. To view a copy of this licence, visit http://creativecommons.org/licenses/by/4.0/.

\section{References}

1. Fölster-Holst R (2014) Management of atopic dermatitis: are there differences between children and adults? J Eur Acad Dermatol Venereol 28(Suppl 3):5-8

2. Mortz CG, Andersen KE, Dellgren C, Barington T, Bindslev-Jensen C (2015) Atopic dermatitis from adolescence to 
adulthood in the toaCS cohort: prevalence, persistence and comorbidities. Allergy 70:836-845

3. Bannister MJ, Freeman S (2000) Adult-onset atopic dermatitis. Australas J Dermatol 41:225-228

4. Shaw TE, Currie GP, Koudelka CW, Simpson EL (2011) Eczema prevalence in the United States: data from the 2003 National Survey of Children's Health. J Invest Dermatol 131:67-73

5. Barbarot S, Auziere S, Gadkari A, Girolomoni G, Puig L, Simpson EL, Margolis DJ, de Bruin-Weller M, Eckert L (2018) Epidemiology of atopic dermatitis in adults: results from an international survey. Allergy 73:1284-1293

6. Guttman-Yassky E, Krueger JG, Lebwohl MG (2018) Systemic immune mechanisms in atopic dermatitis and psoriasis with implications for treatment. Exp Dermatol 27:409-417

7. Kok WL, Yew YW, Thng TG (2019) Comorbidities associated with severity of atopic dermatitis in young adult males: a national cohort study. Acta Derm Venereol 99(7):652-656. https://doi.org/10.2340/00015555-3175

8. Wollenberg A, Oranje A, Deleuran M, Force ET, on Atopic Dermatitis, EADV Eczema Task Force, ETFAD, EADV eczema task force, et al (2015) position paper on diagnosis and treatment of atopic dermatitis in adult and paediatric patients. J Eur Acad Dermatol Venereol 30(2016):729-747. https://doi.org/10. $1111 / j d v .13599$

9. Muraro A, Lemanske RF Jr, Hellings PW et al (2016) Precision medicine in patients with allergic diseases: airway diseases and atopic dermatitis- PRACTALL document of the European Academy of Allergy and Clinical Immunology and the American Academy of Allergy, Asthma \& Immunology. J Allergy Clin Immunol 137:1347-1358. https://doi.org/10.1016/j.jaci.2016.03.010)

10. Hanifin JM, Thurston M, Omoto M, Cherill R, Tofte SJ, Graeber M (2001) The eczema area and severity index (EASI): assessment of reliability in atopic dermatitis. EASI Evaluator Group Exp Dermatol 10(1):11-18

11. Kunz B, Oranje AP, Labrèze L, Stalder JF, Ring J, Taïeb A (1997) Clinical validation and guidelines for the SCORAD index: consensus report of the European Task Force on Atopic Dermatitis. Dermatology 195(1):10-19

12. Patruno C, Amerio P, Chiricozzi A, Costanzo A, Cristaudo A, Cusano F, Foti C, Girolomoni G, Guarneri F, Naldi L, Offidani A, Pigatto P, Prignano F, Stingeni L, Calzavara PP (2020) Optimizing a clinical guidance for diagnosis of atopic dermatitis in adults: joint recommendations of the Italian Society of Dermatology and Venereology (SIDeMaST), Italian Association of Hospital Dermatologists (ADOI), and Italian Society of Allergological, Occupational and Environmental Dermatology (SIDAPA). G Ital Dermatol Venereol 155(1):1-7. https://doi. org/10.23736/S0392-0488.19.06522-2 (Epub 2019 Dec 16)

13. Finlay AY, Khan GK (1994) Dermatology Life Quality Index (DLQI)-a simple practical measure for routine clinical use. Clin Exp Dermatol 19(3):210-216

14. Sicras-Mainar A, Navarro-Artieda R, Armario-Hita JC (2019) Severe Atopic Dermatitis In Spain: A Real-Life Observational
Study. Ther Clin Risk Manag 15:1393-1401. https://doi.org/10. 2147/TCRM.S226456

15. Thijs JL, Strickland I, Bruijnzeel-Koomen CAFM, Nierkens S, Giovannone B, Csomor E et al (2017) Moving toward endotypes in atopic dermatitis: identification of patient clusters based on serum biomarker analysis. J Allergy Clin Immunol 140:730-737

16. Bantz SK, Zhu Z, Zheng T (2014) The atopic march: progression from atopic dermatitis to allergic rhinitis and asthma. J Clin Cell Immunol 5(2):202

17. Hill DA, Spergel JM (2018) The atopic march: critical evidence and clinical relevance. Ann Allergy Asthma Immunol 120(2):131137. https://doi.org/10.1016/j.anai.2017.10.037

18. Gandhi NA, Bennett BL, Graham NM, Pirozzi G, Stahl N, Yancopoulos GD (2016) Targeting key proximal drivers of type 2 inflammation in disease. Nat Rev Drug Discov 15(1):35-50. https://doi.org/10.1038/nrd4624

19. Furue M, Kadono T (2017) "Inflammatory skin march" in atopic dermatitis and psoriasis. Inflamm Res 66(10):833-842. https:// doi.org/10.1007/s00011-017-1065-z (Epub 2017 Jun 15)

20. Hu Y, Zhu Y, Lian N, Chen M, Bartke A, Yuan R (2020) Metabolic syndrome and skin diseases. Front Endocrinol (Lausanne) 10:788. https://doi.org/10.3389/fendo.2019.00788

21. Radtke MA, Schafer I, Glaeske G, Jacobi A, Augustin M (2017) Prevalence and comorbidities in adults with psoriasis compared to atopic eczema. J Eur Acad Dermatol Venereol 31:151-157. https://doi.org/10.1111/jdv.13813

22. Ali Z, Ulrik CS, Agner T, Thomsen SF (2018) Association between atopic dermatitis and the metabolic syndrome: a systematic review. Dermatology 234:79-85. https://doi.org/10.1159/ 000491593

23. Silverberg JI, Silverberg NB, Lee-Wong M (2012) Association between atopic dermatitis and obesity in adulthood. Br J Dermatol 166(3):498-504. https://doi.org/10.1111/j.1365-2133.2011. 10694.x

24. Silverberg JI, Silverberg NB, Lee-Wong M, Zhang A, Silverberg JI (2015) Association of atopic dermatitis with being overweight and obese: a systematic review and meta-analysis. J Am Acad Dermatol 72:606-16e604. https://doi.org/10.1016/j.jaad.2014.12. 013

25. Thyssen JP, Halling-Overgaard AS, Andersen YMF, Gislason G, Skov L, Egeberg A (2018) The association with cardiovascular disease and type 2 diabetes in adults with atopic dermatitis: a systematic review and meta-analysis. Br J Dermatol 178:1272-1279

26. Ascott A, Mulick A, Yu AM, Prieto-Merino D, Schmidt M, Abuabara K et al (2019) Atopic eczema and major cardiovascular outcomes: a systematic review and meta-analysis of population-based studies. J Allergy Clin Immunol 143(5):1821-1829

27. Shah N, Alhusayen R, Walsh S, Shear NH (2018) Methotrexate in the treatment of moderate to severe atopic dermatitis: a retrospective study. J Cutan Med Surg 22(5):484-487

Publisher's Note Springer Nature remains neutral with regard to jurisdictional claims in published maps and institutional affiliations. 


\section{Authors and Affiliations}

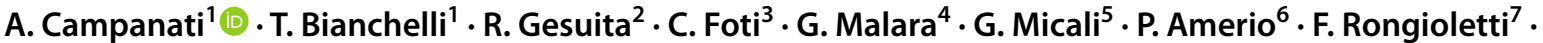 M. Corazza ${ }^{8} \cdot$ A. Patrizi ${ }^{9} \cdot$ K. Peris ${ }^{10,11,12} \cdot$ N. Pimpinelli ${ }^{13}$ - A. Parodi ${ }^{14} \cdot$ M. C. Fargnoli ${ }^{15}$ - S. P. Cannavo ${ }^{16} \cdot$ P. Pigatto $^{17}$. G. Pellacani ${ }^{18}$ - S. M. Ferrucci ${ }^{19,20} \cdot$ G. Argenziano ${ }^{21} \cdot$ F. Cusano ${ }^{22} \cdot$ G. Fabbrocini ${ }^{23} \cdot$ L. Stingeni $^{24} \cdot$ M. C. Potenza ${ }^{25}$. M. Romanelli ${ }^{26} \cdot$ L. Bianchi ${ }^{27} \cdot$ A. Offidani ${ }^{1} \cdot$ and collaborators}

\author{
A. Campanati \\ anna.campanati@gmail.com \\ C. Foti \\ caterina.foti@uniba.it \\ G. Malara \\ giovanna.malara@ospedalerc.it \\ G. Micali \\ gimicali1@hotmail.it \\ P. Amerio \\ p.amerio@unich.it \\ F. Rongioletti \\ rongioletti@unica.it
}

M. Corazza

monica.corazza@unife.it

K. Peris

ketty.peris@policlinicogemelli.it

N. Pimpinelli

nicola.pimpinelli@unifi.it

A. Parodi

aurora.parodi@unige.it

M. C. Fargnoli

mariaconcetta.fargnoli@univaq.it

S. P. Cannavo

serafinella.cannavo@polime.it

P. Pigatto

paolo.pigatto@unimi.it

G. Pellacani

giovanni.pellacani@unimore.it

S. M. Ferrucci

silviaferrucci@hotmail.com

G. Argenziano

giuseppe.argenziano@unicampania.it

F. Cusano

francesco.cusano2@tin.it

G. Fabbrocini

gabriella.fabbrocini@unina.it

L. Stingeni

luca.stingeni@unipg.it

M. C. Potenza

concetta.potenza@uniroma1.it

M. Romanelli

m.romanelli@med.unipi.it

L. Bianchi

luca.bianchi@uniroma2.it
A. Offidani

a.offidani@ospedaliriuniti.marche.it

1 Department of Clinical and Molecular Sciences,

Dermatology Unit, Polytechnic Marche University, Ancona, Italy

2 Centre of Epidemiology and Biostatistics, Polytechnic Marche University, Ancona, Italy

3 Department of Biomedical Science and Human Oncology, Dermatological Clinic, University of Bari, Bari, Italy

4 Department of Dermatology Grande, Ospedale Metropolitano "Bianchi Melacrino Morelli", Reggio Calabria, Italy

5 Dermatology Clinic, University of Catania, PO G. Rodolico, AOU Policlinico-Vittorio Emanuele, Catania, Italy

6 Department of Medicine and Aging Science, Dermatology Unit, University G.D’Annunzio Chieti, Chieti, Italy

7 Vita-Salute - S, Raffaele University, Milan, Italy

8 Department of Medical Sciences, Dermatology Unit, University of Ferrara, Ferrara, Italy

9 Department of Experimental, Diagnostic and Specialty Medicine, Division of Dermatology, University of Bologna, Bologna, Italy

10 Fondazione Policlinico Universitario A. Gemelli IRCCS, Dermatologia, Rome, Italy

11 Sezione di Dermatologia, Dipartimento di Scienze Mediche e Chirurgiche, Università Cattolica del Sacro Cuore, Rome, Italy

12 Oncologia Medica, Università Cattolica del Sacro Cuore, Rome, Italy

13 Department Health Science Section of Dermatology, University of Florence, Florence, Italy

14 Dermatology Clinic, Ospedale Policlinico San Martino, Largo R. Benzi, 10, 16132 Genoa, Italy

15 Dermatology, Department of Biotechnological and Applied Clinical Sciences, University of L'Aquila, L'Aquila, Italy

16 Dermatology Unit, University Hospital Policlinico "G. Martino", Messina, Italy

17 Clinical Dermatology, IRCCS Istituto Ortopedico Galeazzi, Milan, Italy

18 Dermatology Unit, Department of Surgical, Medical, Dental and Morphological Science With Interest Transplant, Oncological and Regenerative Medicine, University of Modena and Reggio Emilia, Modena, Italy

19 Department of Physiopathology and Transplantation, Università Degli Studi Di Milano, Milan, Italy 
20 Dermatology Unit, Fondazione IRCCS Ca' Granda Ospedale Maggiore Policlinico, Via Pace, 9, 20122 Milan, Italy

21 Dermatology Unit, University of Campania, Naples, Italy

22 Unit of Dermatology, G. Rummo Hospital, Benevento, Italy

23 Department of Clinical Medicine and Surgery, Section of Dermatology, University of Naples Federico II, Naples, Italy

24 Dermatology Section, Department of Medicine, University of Perugia, Perugia, Italy
25 Dermatology Unit, Department of Medico-Surgical Sciences and Biotechnologies, Faculty of Pharmacy and Medicine, Sapienza University of Rome - Polo Pontino, Rome, Italy

26 Dermatology Unit, Department of Clinical and Experimental Medicine, University of Pisa, Pisa, Italy

27 Dermatology Unit, Policlinico Tor Vergata Rome, University of Rome Tor Vergata, Rome, Italy 